\title{
Recent Progress in Lorentz Transmission Electron Microscopy
}

\section{Applications to Multi-Ferroic Materials}

\author{
Marc De Graef ${ }^{a}$ \\ Department of Materials Science and Engineering, Carnegie Mellon University, 5000 Forbes Avenue, \\ Pittsburgh PA 15213-3890, USA
}

\begin{abstract}
After a brief review of the basic methods of Lorentz transmission electron microscopy (LTEM), including the Transport-of-Intensity formalism for phase reconstruction, we present a few examples of the application of LTEM to multiferroic materials, in this case ferromagnetic shape memory alloys. We discuss observations of magnetic domain walls pinned to anti-phase boundaries in $\mathrm{Ni}_{2} \mathrm{MnGa}$, and domain wall behavior under an in-situ applied magnetic field in Fe-Pd-Co.
\end{abstract}

\section{Lorentz Microscopy: a Brief Introduction}

It is one of the primary goals of transmission electron microscopy (TEM) to determine quantitatively the structure and chemistry of solids. For a periodic structure, this involves determination of the content of the unit cell (atom types and positions). For magnetic materials, this includes determination of the local magnetization state. The characterization of magnetic microstructures is a complex problem, since it involves, in principle, the determination of a three-dimensional (3-D) vector field, i.e., the magnetization $\mathbf{M}(\mathbf{r})$, or the induction $\mathbf{B}(\mathbf{r})$.

Lorentz TEM (or LTEM) relies on the fact that a high energy beam electron is deflected by the magnetic induction inside and around the sample. The deflection angle for a uniformly magnetized foil can be computed from a simple momentum balance [1] and is given by $\theta_{L}=$ $C_{L} B_{\perp} t$, with $C_{L}=e \lambda / h, \lambda$ the electron wavelength, $B_{\perp}$ the induction component normal to the beam, and $t$ the foil thickness. This beam deflection is usually about two orders of magnitude smaller than a typical Bragg angle, which means that the magnetization state in a foil must be studied by analyzing the fine-structure or splitting of the transmitted beam. The primary method to detect small deflections is to defocus the imaging lens, i.e., to look at a plane below or above the sample, so that the lateral shift of the deflected electrons can be measured; the larger the defocus, the larger the lateral shift and, hence, the easier the measurement. However, the larger the defocus, the more blurred the resulting image becomes, so that there is a practical limit to the amount of defocus that will produce interpretable magnetic contrast.

The Lorentz deflection angle can be converted into a magnetic phase gradient [2] by means of the relation $\nabla \varphi_{m}=2 \pi k \theta_{L} \mathbf{e}$, where $k=1 / \lambda$ and $\mathbf{e}$ is a unit vector. Integrating this relation and substituting typical numerical values shows that magnetic films should normally be regarded as strong, albeit slowly varying, phase objects. It was shown by Aharonov and Bohm [3] that the total phase shift of an electron wave traveling through a magnetic material consists of two contributions $\left(\mathbf{r}_{\perp}\right.$ is a vector in the plane normal to the beam):

$$
\varphi\left(\mathbf{r}_{\perp}\right)=\varphi_{e}\left(\mathbf{r}_{\perp}\right)+\varphi_{m}\left(\mathbf{r}_{\perp}\right)=C_{E} \int_{-\infty}^{+\infty} V\left(\mathbf{r}_{\perp}+\ell \hat{\boldsymbol{\omega}}\right) \mathrm{d} \ell-C_{B} \int_{-\infty}^{+\infty} \mathbf{A}\left(\mathbf{r}_{\perp}+\ell \hat{\boldsymbol{\omega}}\right) \cdot \hat{\boldsymbol{\omega}} \mathrm{d} \ell .
$$

\footnotetext{
a e-mail: degraef@cmu.edu
} 
The constants are given by $C_{E}=\pi / E \lambda$, with $E$ the relativistic electron accelerating potential [4] and $C_{B}=e / \hbar$. Integration occurs along the beam direction $\hat{\boldsymbol{\omega}}$ and is parametrized by $\ell$. $V(\mathbf{r})$ is the electrostatic potential of the sample, and $\mathbf{A}(\mathbf{r})$ the magnetic vector potential.

The objective lens of a TEM is an immersion lens, meaning that the sample sits inside the magnetic field of the lens. At axial field strengths of more than $2 \mathrm{~T}$, most materials are saturated by the lens field, so that LTEM observations are usually carried out with the main objective lens turned off; a weaker lens, located a few centimeters below the sample, is used to focus the electron beam. Such a Lorentz lens is generally characterized by a spherical aberration constant, $C_{s}$, in the range 100-10000 mm, compared to values in the range of $0.5-1.0 \mathrm{~mm}$ for a state-of-the-art TEM operated in normal mode. The $200 \mathrm{kV}$ Tecnai F20, located in the Materials Characterization Laboratory (MCL) at CMU, has a spherical aberration constant of 7.4 meters in Lorentz mode. At a Scherzer defocus of $\Delta f=\left(3 C_{s} \lambda / 2\right)^{1 / 2}=5276 \mathrm{~nm}$, the Scherzer point resolution is given by $\rho_{S}=0.64\left(C_{s} \lambda^{3}\right)^{1 / 4}=2.1 \mathrm{~nm}$ [1]. The small Lorentz deflection angle, however, typically necessitates a much larger defocus value in order to reveal magnetic contrast; a defocus measured in the tens or hundreds of microns is not uncommon. This, in turn, results in significant image blurring, limiting the spatial resolution to about 10 $\mathrm{nm}$. A reduced $C_{s}$ in LTEM mode would allow smaller defocus values to be employed, thereby improving the spatial resolution. This will be addressed briefly in section 3 .

There are two experimental methods to determine the phase, $\varphi$, of the electron wave: electron holography [5] and the Transport-of-Intensity Equation (TIE) formalism [6]. The TIE formalism relies on the following equation for the phase $\varphi\left(\mathbf{r}_{\perp}\right)$ of the electron wave:

$$
\nabla_{\perp} \cdot\left[I\left(\mathbf{r}_{\perp}, 0\right) \nabla_{\perp} \varphi\left(\mathbf{r}_{\perp}\right)\right]=-\left.\frac{2 \pi}{\lambda} \frac{\partial I\left(\mathbf{r}_{\perp}, z\right)}{\partial z}\right|_{z=0}
$$

where $I\left(\mathbf{r}_{\perp}, z\right)$ is the image intensity at defocus plane $z, \mathbf{r}_{\perp}$ a $2 \mathrm{D}$ position vector in this plane, and $\lambda$ the electron wavelength; $\nabla_{\perp}$ is the $2 \mathrm{D}$ gradient operator. The right-hand side derivative in this equation can be determined numerically from a through-focus series of Lorentz images. The phase $\varphi$ is then determined by solving the equation by means of fast Fourier transforms $[1,7]$. This method is exact when the defocus is kept small [8].

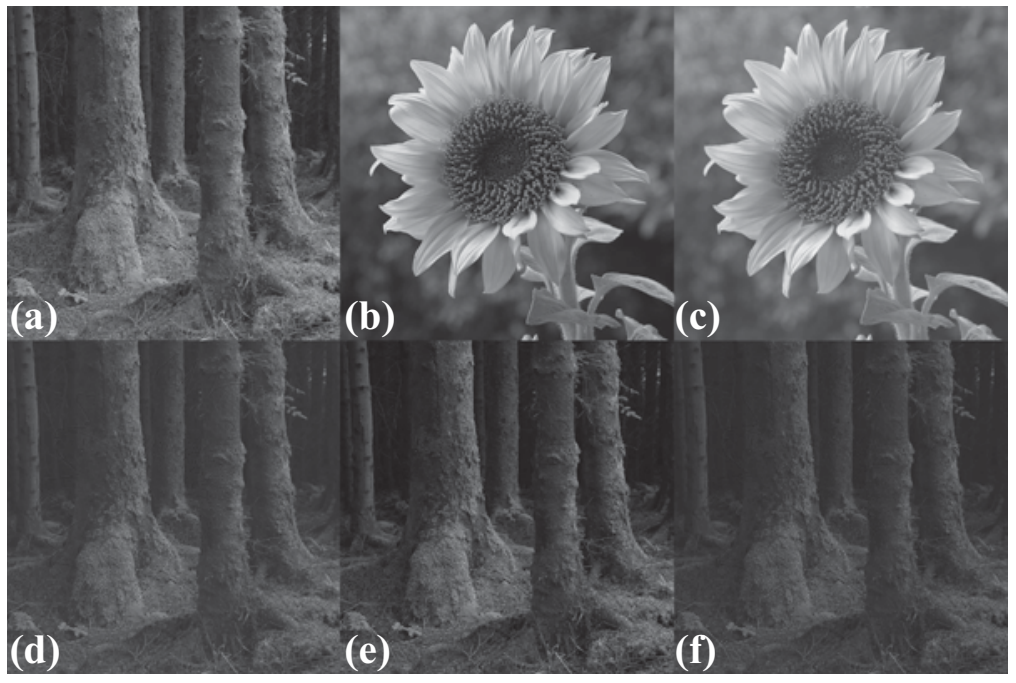

Fig. 1. (a) and (b) are amplitude and phase images (both images are royaltyfree public domain images from http://www.publicdomain-image.com) used to compute the through focus series in (d, $z=-10$ $\mu \mathrm{m}),(\mathrm{e}, z=0)$, and (f, $z=+10 \mu \mathrm{m})$. The phase reconstructed from the through-focus series is shown in (c); note the slight blurring of sharp edges compared to the input phase in (b).

To illustrate the use of the TIE formalism, consider the following simple example. We construct an object exit wave using two public domain images, one for the amplitude $a\left(\mathbf{r}_{\perp}\right)$ of the wave, the other for the phase, $\varphi\left(\mathbf{r}_{\perp}\right)$, as shown in Fig. 1(a) and (b). We assign a length scale of $1 \mathrm{~nm} /$ pixel and construct the wave function $\psi\left(\mathbf{r}_{\perp}\right)=a\left(\mathbf{r}_{\perp}\right) \exp \left[\mathrm{i} \varphi\left(\mathbf{r}_{\perp}\right)\right]$. The LTEM image formation process can be expressed in terms of a partially coherent contrast transfer function 
$(\mathrm{CTF}), T(q)$, in reciprocal space, given by [1]:

$$
T(q)=e^{-\left(\gamma_{r}+\mathrm{i} \gamma_{i}\right) q^{2}} \quad \text { with } \quad \gamma_{r}=\left(\pi \theta_{c} z\right)^{2} \quad \text { and } \quad \gamma_{i}=-\pi \lambda z
$$

$z$ is the defocus, and $\theta_{c}$ the beam divergence angle, typically $10^{-5} \mathrm{rad}$ for a field emission electron gun. Other lens aberrations, such as spherical aberration, are ignored for this example. Fig. 1(d) through (f) show the computed images for $\theta_{c}=10^{-5} \mathrm{rad}$ and $z= \pm 10 \mu \mathrm{m}$; the central image $(z=0)$ shows only amplitude contrast, whereas in the out-of-focus images amplitude and phase contrast are mixed. The outline of the flower is weakly visible in the out-of-focus images. After substituting the difference of the out-of-focus images as the right-hand side of eq. (2), the phase can be reconstructed (Fig. 1(c)). Note that sharp edges are somewhat blurred in the reconstruction, a consequence of the low-pass filter characteristics of the numerical process. In section 2, we will show several examples of the use of the TIE formalism on real LTEM images.

\section{Applications to Ferromagnetic Shape Memory Alloys}

In this section, we provide examples of the application of LTEM to multiferroic materials. The materials used for this study are:

- $\mathrm{Fe}_{68} \mathrm{Pd}_{30} \mathrm{Co}_{2}$, arc melted, heat treated for three days at $1100^{\circ} \mathrm{C}$, then three hours at $90^{\circ} \mathrm{C}$ followed by a water quench. At room temperature, the sample state is martensitic with a very low tetragonality, effectively a bcc martensite with $a=0.2976 \mathrm{~nm}$. The $2 \times 2 \mathrm{~mm}$ square thin foil was electropolished at $0^{\circ} \mathrm{C}$ and $15 \mathrm{~V}$ in a $82 \%$ acetic acid, $9 \%$ ethanol, $9 \%$ perchloric acid electrolyte, and subsequently mounted in a Hummingbird Scientific magnetic field stage in a dedicated JEOL 2100F Lorentz TEM operated at $200 \mathrm{kV}$.

- $\mathrm{Ni}_{50} \mathrm{Mn}_{27} \mathrm{Ga}_{23}$, arc melted, heat treated for two days at $800^{\circ} \mathrm{C}$ and water quenched. At room temperature, the sample is mostly austenitic (cubic, with $a=0.582 \mathrm{~nm}$ ) with a dense anti-phase boundary network. The TEM thin foil was jet polished at $-40^{\circ} \mathrm{C}$ at $15 \mathrm{~V}$ in $95 \%$ methanol, 5\% perchloric and studied in a JEOL 2100F Lorentz TEM operated at $200 \mathrm{kV}$.

We describe the results of an in-situ magnetic field study of FePdCo in section 2.1, and a study of domain wall pinning on anti-phase boundaries in $\mathrm{Ni}_{2} \mathrm{MnGa}$ is presented in section 2.2.

\subsection{Magnetic domain structures under an applied in-situ magnetic field in FePdCo}

Since in LTEM the sample is located in a field-free environment, it becomes possible to subject it to a magnetic field applied perpendicular to the electron beam. Using a Hummingbird Scientific in-situ magnetizing sample holder, we have studied an $\mathrm{Fe}_{68} \mathrm{Pd}_{30} \mathrm{Co}_{2}$ sample in the martensitic state. As shown in Fig. 2(a), at zero applied field there are two types of magnetic domain walls: those that are pinned by martensite plate boundaries (white arrows) and those that are mobile (black arrows). The field-of-view of this image is $8.65 \mu \mathrm{m}^{2}$.

In (b), an in-plane field of 107 Oe has been applied; although the magnetizing holder is capable of generating much larger fields, this is the maximum field that can be applied for which the beam deflection can still be corrected by means of the microscope's deflection coils. Note that the mobile walls have disappeared, and the pinned walls are still present (white arrows). When the applied field is reduced, the mobile walls (black arrows) reappear in (c) $(+6$ $\mathrm{Oe})$ and $(\mathrm{d})(-5.3 \mathrm{Oe})$. After reversing the applied field, a magnetization reversal has occurred between (e) ( $-16.5 \mathrm{Oe})$ and (f) $(-27.8 \mathrm{Oe})$; this is clearly observed via the reversal of domain wall contrast (arrowed locations). It is anticipated that more powerful beam deflectors will allow for the study of field-induced variant boundary motion in muti-ferroic materials with a low boundary motion energy. 


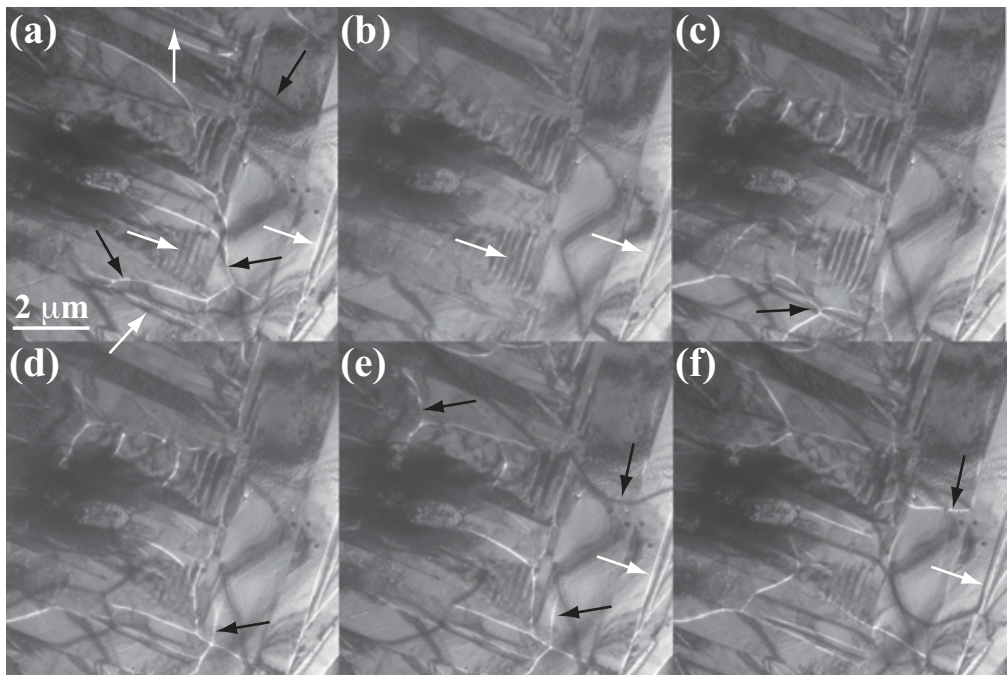

Fig. 2. A series of LTEM images of martensite plates in a FePdCo alloy. The sample is at zero applied magnetic field in (a); arrows indicate mobile (black) and pinned (white) domain walls. In (b), the sample is exposed to the largest field (107 Oe) possible in this experimental setup. The field is reduced in (c) and (d), which are taken around zero applied field. (e) and (f) show the effect of a magnetization reversal. See text for additional information.

\subsection{Pinning of magnetic domain walls on anti-phase boundaries in $\mathrm{Ni}_{2} \mathrm{MnGa}$}

We have reported previously that anti-phase boundaries (APBs) in $\mathrm{Ni}_{2} \mathrm{MnGa-type} \mathrm{alloys} \mathrm{are} \mathrm{not}$ visible by means of standard diffraction contrast observations due to a rather large extinction distance for the superlattice reflections [9]. Typically, a high density of APBs is present in quenched alloys; when these are analyzed by means of LTEM, nearly all APBs appear to be decorated with magnetic domain walls that are pinned by the local change in magnetization that is associated with the local imperfection in the ordering of the Mn sub-lattice.

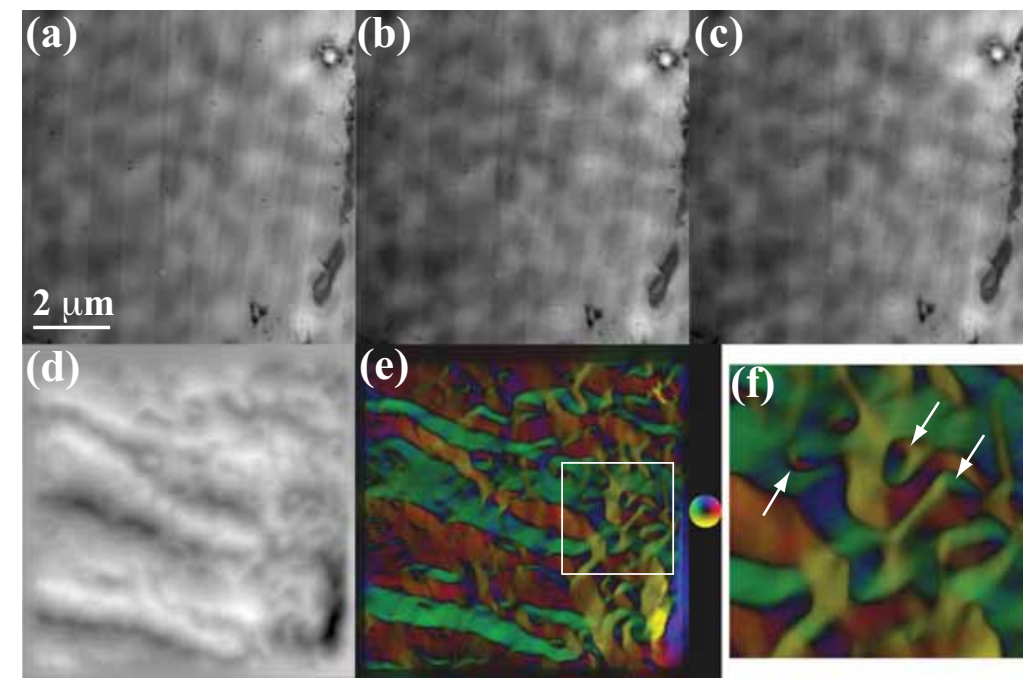

Fig. 3. In-focus image (a), under-focus (b), and over-focus (c) images of a martensitic region in a water-quenched $\mathrm{Ni}_{50} \mathrm{Mn}_{27}$ $\mathrm{Ga}_{23}$ alloy. (d) shows the reconstructed exit wave phase shift as a grayscale map. In (e), the gradient of the phase map is represented as a color-coded map; the color indicates the direction of the local (integrated) magnetic induction. Note the presence of multiple magnetization vortices in the magnified portion (f).

A through-focus series of images of $\mathrm{Ni}_{50} \mathrm{Mn}_{27} \mathrm{Ga}_{23}$ in the martensitic state is shown in Fig. 3(a)-(c). The mottled contrast in (a) is due to sample thickness variations caused by imperfect electrolytic polishing, and the nearly vertical lines correspond to surface relief left over on the thin foil due to the presence of martensite plates at the temperature at which the thin foil was electrolytically thinned. In the out-of-focus images the magnetic domain walls are clearly visible and they exhibit a pattern reminiscent of that of APBs with isotropic boundary energy. Using the TIE formalism, the electron wave phase shift was reconstructed (d), and from the phase shift gradient a projected (integrated) induction map was computed (e); i.e, the gradient 
of the phase shift is proportional to the integral of the magnetic induction along the beam electron trajectory. The color corresponds to the local orientation of the magnetic induction. (f) is a magnified portion of (e), showing a number of magnetization vortices (arrowed). Note that the magnetic domain walls appear to be unaffected by the martensite plate boundaries.

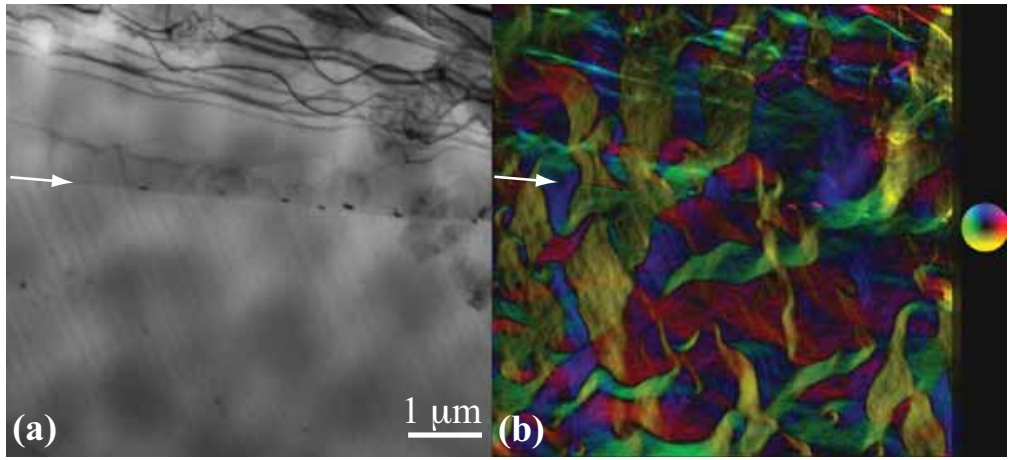

Fig. 4. (a) in-focus image of the boundary between austenite and martensite (arrowed). In (b), the integrated induction map is shown along with a color wheel. Both $180^{\circ}$ and $90^{\circ}$ domain walls can be observed, with the former mostly across anti-phase boundaries, and the latter in between the APBs.

Fig. 4(a) shows another example of interactions between domain walls and microstructural features, in this case a boundary (arrowed) decorated with small precipitates between an austenitic region (top) and a finely twinned martensitic region (bottom). From the out-of-focus images (not shown) the exit wave phase is reconstructed, and its gradient is shown in (b). Note that the domain walls again are coincident with anti-phase boundaries. Most APBs are associated with $180^{\circ}$ domain walls, which can be derived from the fact that the colors on either side of the APBs lie on opposite sides of the color wheel. The regions in between two APBs generally show alternating colors that are only $90^{\circ}$ apart on the color wheel, indicating the presence of numerous $90^{\circ}$ walls. The domain wall contrast does not appear to be affected by the presence of the boundary (arrowed); most domain walls continue uninterruptedly across the boundary.

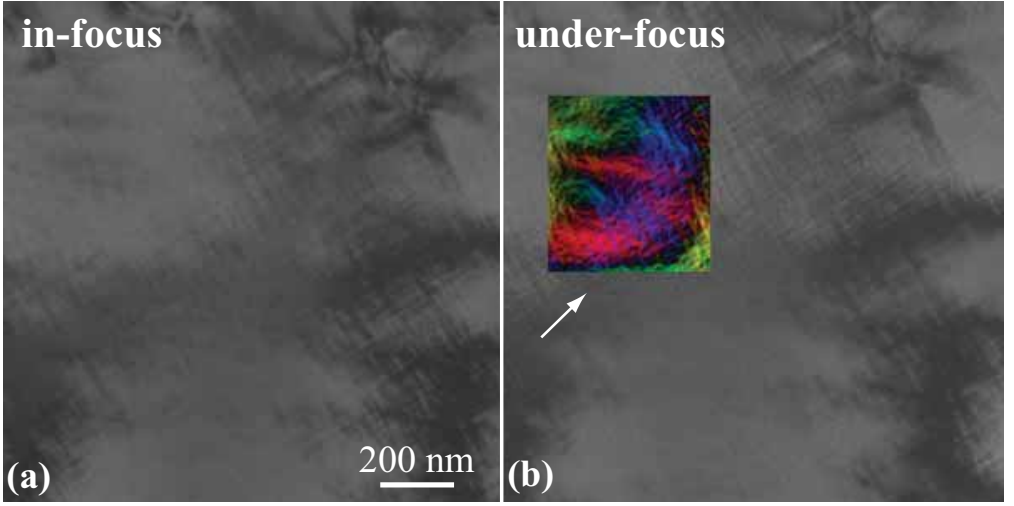

Fig. 5. (a) in-focus and (b) under-focus images of a region near a bend contour in a $\mathrm{Ni}_{2} \mathrm{MnGa}$ thin foil. The contrast modulations in (a) are known as structural tweed; the additional modulations in (b) correspond to the presence of magneto-elastic modulations due to the strong coupling of magnetic and elastic effects in this alloy.

A final example of magnetic contrast in $\mathrm{Ni}_{2} \mathrm{MnGa-type}$ alloys is shown in Fig. 5: (a) is an infocus image of traditional structural tweed contrast near a bend contour (a strongly diffracting region in the thin foil). The image in (b) is the under-focus version of (a); note that the tweed contrast is significantly enhanced with respect to the in-focus image, and that tweed contrast is visible in regions where the in-focus images does not show any tweed contrast. This additional contrast is a signature of "magneto-elastic tweed," i.e., a modulation of the local magnetization. The integrated induction map in (b) employs the same color wheel legend as in previous figures. Note the fine-scale magnetic modulations with a periodicity of around $20-30 \mathrm{~nm}$. The arrow indicates a single APB, visible as a faint line on top of the modulated background. Theoretical models for this magneto-elastic tweed are consistent with the type of contrast observed, but the 
resolution of the LTEM mode used to obtain these images is, at present, insufficient to provide additional details about the nature of the magneto-elastic tweed [10].

\section{Some Remarks on the Future of LTEM and Summary}

In recent years, aberration-corrected transmission electron microscopes have become available commercially. These instruments allow for the correction of spherical aberration, which is the main objective lens aberration, in particular for Lorentz microscopy, where it is not uncommon to encounter lenses with several hundred to several thousand millimeters of spherical aberration. For comparison, in high-resolution imaging mode, this aberration is typically measured to be around $0.5 \mathrm{~mm}$, and with correction it can be made to vanish entirely. The resolution of a Lorentz lens can be improved significantly by bringing the spherical aberration down to just a few millimeters, which translates into a spatial resolution of better than $0.5 \mathrm{~nm}$.

Correction of chromatic aberration is now also possible [11] and this will result in instruments that produce images with a vastly improved signal-to-noise ratio, both in conventional and Lorentz imaging modes. With special objective lens designs, it will become possible in the near future to have both spherical and chromatic aberration corrected with a field free sample environment; this should allow for simultaneous high resolution and Lorentz imaging. It should also become possible to tune the chromatic aberration corrector so that it effectively becomes a phase plate; this would allow for the direct observation of the electrostatic and magnetic phase shifts caused by the sample, without the need for numerical reconstruction.

Recent advances in tomography are also having an impact on the characterization of magnetic materials. It is now possible to determine the three-dimensional magnetic vector potential of a magnetic object at a length scale below one micrometer. Once the vector potential is known, all other magnetic quantities of interest can be derived by simple differentiation.

In the present paper, we have given a brief overview of recent experimental results in the area of magnetic domain characterization in ferromagnetic shape memory alloys. With the aid of phase reconstruction, it has become possible to determine quantitatively how magnetic domains interact with other crystallographic features, such as APBs and twin boundaries.

The author would like to thank several current and former students and colleagues for stimulating interactions: S. Tandon, C. Phatak, S.P. Venkateswaran, A. Budruk, M. Wuttig, A. Petford-Long, B. Kabius, D. Laughlin, M. McHenry, and N.T. Nuhfer. Financial support from the National Science Foundation and the US Department of Energy is also acknowledged.

\section{References}

1. M. De Graef. Lorentz microscopy: Theoretical basis and image simulations. In M. De Graef and Y. Zhu, editors, Magnetic Microscopy and its Applications to Magnetic Materials, volume 36 of Experimental Methods in the Physical Sciences, Academic Press, 2000, Chapter 2.

2. J.N. Chapman, E.M. Waddell, P.E. Batson, and R.P. Ferrier. Ultramicroscopy, 4 (1979) 283-292.

3. Y. Aharonov and D. Bohm. Phys. Rev., 115 (1959) 485-491.

4. M. De Graef. Introduction to Conventional Transmission Electron Microscopy. (Cambridge University Press, Cambridge 2003).

5. E. Völkl and M. Lehmann. The reconstruction of off-axis electron holograms. In E. Völkl, L.F. Allard, and D.C. Joy, editors, Introduction to Electron Holography, (Kluwer Academic, New York 1999), pages $125-151$.

6. D. Paganin and K.A. Nugent. Phys.Rev.Lett., 80 (1998) 2586-2589.

7. V.V. Volkov, Y. Zhu, and M. De Graef. Micron, 33 (2002) 411-416.

8. M. Beleggia, M.A. Schofield, V.V. Volkov, and Y. Zhu. Ultramicroscopy, 102 (2002) 37-49.

9. S.P. Venkateswaran, N.T. Nuhfer, and M. De Graef. Acta Materialia, 55 (2007) 2621-2636.

10. A. Saxena, T. Castán, A. Planes, M. Porta, Y. Kishi, T.A. Lograsso, D. Viehland, M. Wuttig, and M. De Graef. Phys. Rev. Lett., 92 (2004) 197203.

11. M. Haider, H. Müller, S. Uhlemann, J. Zach, U. Loebau, and R. Hoeshen. Ultramicroscopy, 108 (2008) 167-178. 